\title{
Levantamento epidemiológico das lesões bucais nos pacientes atendidos nas clínicas da Faculdade de Odontologia da Universidade de Passo Fundo
}

\section{Epidemiological survey of oral lesions in patients assisted at the clinics of the School of Dentistry of the University of Passo Fundo}

Karen Hoff

Soluete Oliveira da Silva**

João Paulo De Carli**

\section{Resumo}

A importância da realização de estudos epidemiológicos, além de determinar a prevalência de lesões encontradas em uma determinada área geográfica, é estabelecer métodos preventivos e realizar promoção da saúde bucal. Objetivo: o objetivo deste trabalho é efetuar um levantamento epidemiológico das lesões bucais mais frequentes com base nos prontuários odontológicos da Faculdade de Odontologia da Universidade de Passo Fundo (FOUPF), entre os anos 2000 e 2013. Materiais e método: foram examinados 18.801 prontuários. Dos dados coletados, 940 lesões foram encontradas e analisadas com o programa SPSS, versão 15.0. Resultados: as cinco lesões mais prevalentes foram fibroma de irritação $(15,1 \%)$, candidíase $(7,6 \%)$, hiperplasia fibrosa dos sul$\cos (7,3 \%)$, mucocele $(7,2 \%)$ e líquen plano $(5,5 \%)$. O gênero feminino foi o mais acometido (6,5\%), a etnia mais prevalente, a leucoderma (90\%). O grupo das lesões proliferativas hiperplásicas foi o mais prevalente (29\%), seguido das lesões brancas não ceratóticas (14,9\%), lesões vermelhas da mucosa bucal $(11,3 \%)$, lesões brancas não ceratóticas (9\%) e lesões proliferativas neoplásicas benignas ( $8,3 \%)$. Constatou-se que a faixa etária entre 40 e 69 anos foi a mais acometida $(61,2 \%)$. Conclusão: com a presente pesquisa observou-se que, pelo fato de a FOUPF estar em área geográfica de fácil acesso e ser referência em saúde, tanto médica como odontológica, a procura por atendimento foi de grande importância para as regiões Norte e noroeste do Rio Grande do Sul, possibilitando assim traçar um perfil epidemiológico dos pacientes mais acometidos por lesões bucais.

Palavras-chave: Medicina bucal. Epidemiologia. Lesões bucais.

\section{Introdução}

Os estudos epidemiológicos têm a função de determinar a prevalência e a incidência de inúmeras doenças, e particularizam sua distribuição em características próprias do ambiente onde estão sendo executados $^{1,2}$. Na odontologia, estudos epidemiológicos sobre lesões do complexo bucomaxilofacial são extremamente importantes, principalmente na área estomatológica, pois auxiliam os profissionais na elaboração de hipóteses diagnósticas, fornecendo-lhes dados sobre a prevalência de alterações e doenças, permitindo-lhes então estimar a possibilidade de encontrá-las na sua prática clínica.

Suzin et al. ${ }^{3}$ (2014) analisaram 5.928 prontuários de portadores de patologias de língua no Servi-

Especialista em Patologia Bucal, doutora em Estomatologia Clínica, professora, Faculdade de Odontologia, Universidade de Passo Fundo, Passo Fundo, RS, Brasil.

Especialista em Prótese Dentária, doutor em Estomatologia, professor, Faculdade de Odontologia, Universidade de Passo Fundo, Passo Fundo, RS, Brasil. 
ço de Estomatologia e Prevenção do Câncer do Hospital São Lucas, da Pontifícia Universidade Católica do Rio Grande do Sul, totalizando 7.862 lesões. Das fichas clínicas avaliadas, $41,65 \%$ das enfermidades ocorreram em homens e $58,35 \%$ acometeram mulheres. A maior parte dos casos se concentrou na faixa etária variando entre 40 e 60 anos de idade (40\% dos casos). Dentre as patologias específicas, as línguas saburrosa, fissurada e geográfica foram as doenças que obtiveram o maior número de casos diagnosticados nesse grupo, perfazendo, respectivamente, percentuais de $31,64 \%, 23,98 \%$ e $20,59 \%$. Nas lesões inflamatórias, a hiperplasia fibroepitelial computou 475 casos $(52,26 \%)$, e na faixa etária de 40 a 59 anos (45\%) predominou o granuloma piogênico com 37 casos $(4,07 \%)$. Com relação às neoplasias benignas, o hemangioma foi a lesão mais diagnosticada, somando 146 casos $(52,71 \%)$.

Em um estudo analítico de caráter retrospectivo no qual foi observada a concordância entre o diagnóstico clínico e o histopatológico de pacientes assistidos pela Clínica de Diagnóstico Bucal do Departamento de Odontologia das Faculdades Unidas do Norte de Minas, na cidade de Montes Claros, MG, Souza et al. ${ }^{4}$ (2014) analisaram os pacientes que realizaram biópsia e que tiveram seus laudos histopatológicos emitidos entre fevereiro de 2010 e julho de 2012. A média de idade dos 125 pacientes que atenderam aos critérios de inclusão do estudo e tiveram seus prontuários analisados foi de 48,64 anos, sendo a idade mínima de 6 anos e a máxima de 89 anos. A maior parte dos pacientes era do sexo feminino $(58,4 \%)$ e leucoderma $(36,6 \%)$. Os diagnósticos histopatológicos mais prevalentes pertencem ao grupo dos processos proliferativos não neoplásicos (36,6\%), seguido das lesões cancerizáveis $(16,3 \%)$, neoplasias epiteliais malignas $(10,6 \%)$, doenças autoimunes $(8,1 \%)$ e neoplasias benignas $(1,6 \%)$.

Tendo em vista o exposto, o presente estudo tem como objetivo realizar um levantamento epidemiológico das lesões bucais mais frequentes em pacientes atendidos nas clínicas da FOUPF, com o propósito de traçar um perfil epidemiológico dos portadores, com vistas a estabelecer medidas preventivas futuras.

\section{Materiais e método}

O projeto foi encaminhado ao Comitê de Ética em Pesquisa da Universidade de Passo Fundo e aprovado pelo CAAE nº 20962813.5.0000.5342. Então, foi realizado um levantamento epidemiológico tendo como amostra os prontuários odontológicos das clínicas da FOUPF, analisados a partir do ano 2000 até o ano 2013. Os dados apurados foram registrados em uma planilha de coleta desenvolvida no programa Microsoft Excel ${ }^{\circledR}$, contendo as variáveis a serem estudadas, como o número do prontuário, a idade, o gênero, a etnia, o diagnóstico clínico, a localidade de origem do paciente e a classificação da lesão. Após a compilação, todos os dados foram inseridos no programa SPSS (versão 15.0) e submetidos à análise estatística.

\section{Resultados}

No presente estudo foram analisados 18.801 prontuários odontológicos, dentre os quais foram encontradas 940 lesões estomatológicas. Quanto ao gênero, observou-se que o mais acometido foi o feminino, com 606 casos $(64,5 \%)$, e em relação ao masculino, houve 334 casos $(35,5 \%)$ (Tabela 1). Em relação à etnia, a leucoderma foi a mais prevalente, com $90 \%$ (846 casos), e a melanoderma atingiu $10 \%$ (94 casos) (Tabela 1).

Quanto à idade dos pacientes da amostra estudada, foi observado que variou de 1 a 88 anos, com média de 47,55 anos. Observou-se que a maior parte dos pacientes $(61,2 \%)$ tinha entre 40 e 69 anos de idade. A faixa etária de menor prevalência foi a de 80 a 88 anos, com $0,7 \%$ ( $\mathrm{n}=7$ pacientes) (Tabela 1$)$.

Tabela 1 - Distribuição da amostra estudada segundo faixa etária, gênero e etnia

\begin{tabular}{|c|c|c|c|c|c|c|}
\hline \multirow{2}{*}{ Faixa etária } & \multirow{2}{*}{ № } & \multirow{2}{*}{$\%$} & \multicolumn{2}{|c|}{ Gênero } & \multicolumn{2}{|c|}{ Etnia } \\
\hline & & & Feminino & Masculino & Leucoderma & Melanoderma \\
\hline Menor de 10 anos & 34 & 3,6 & 16 & 18 & 30 & 4 \\
\hline $10-19$ anos & 76 & 8,1 & 43 & 33 & 68 & 8 \\
\hline $20-29$ anos & 64 & 6,8 & 40 & 24 & 59 & 5 \\
\hline $30-39$ anos & 90 & 9,6 & 58 & 32 & 83 & 7 \\
\hline $40-49$ anos & 196 & 20,9 & 132 & 64 & 182 & 14 \\
\hline $50-59$ anos & 209 & 22,2 & 138 & 71 & 176 & 33 \\
\hline $60-69$ anos & 170 & 18,1 & 110 & 60 & 157 & 13 \\
\hline $70-79$ anos & 94 & 10 & 66 & 28 & 85 & 9 \\
\hline Acima de 80 anos & 7 & 0,7 & 3 & 4 & 6 & 1 \\
\hline Total & 940 & 100 & $606(64,5 \%)$ & $334(35,5 \%)$ & $846(90 \%)$ & $94(10 \%)$ \\
\hline
\end{tabular}

Fonte: todas as tabelas são de elaboração dos autores com base nos dados da pesquisa. 
Na Tabela 2, observa-se a distribuição das lesões segundo sua classificação. Dentre os grupos de lesões, as proliferativas hiperplásicas foram as mais frequentes, com $29 \%$, seguidas pelas lesões brancas ceratóticas da mucosa bucal, com $14,9 \%$, lesões vermelhas da mucosa bucal, com $11,3 \%$, lesões brancas não ceratóticas da mucosa bucal, com $9 \%$, e lesões proliferativas neoplásicas benignas, com $8,3 \%$.

Tabela 2 - Distribuição da frequência das lesões estudadas segundo sua classificação

\begin{tabular}{|c|c|c|}
\hline Classificação & $\begin{array}{l}\text { Frequência } \\
\text { (n) }\end{array}$ & $\%$ \\
\hline Alterações de desenvolvimento & 20 & 2,2 \\
\hline $\begin{array}{l}\text { Doenças vesículobolhosas } \\
\text { associadas a defeitos imunológicos }\end{array}$ & 9 & 1,0 \\
\hline $\begin{array}{l}\text { Lesões brancas ceratóticas da } \\
\text { mucosa bucal }\end{array}$ & 140 & 14,9 \\
\hline $\begin{array}{l}\text { Lesões brancas não-ceratóticas da } \\
\text { mucosa bucal }\end{array}$ & 85 & 9,0 \\
\hline Lesões císticas extraósseas & 81 & 8,6 \\
\hline Lesões císticas intraósseas & 9 & 1,0 \\
\hline Lesões proliferativas hiperplásicas & 273 & 29,0 \\
\hline $\begin{array}{l}\text { Lesões proliferativas neoplásicas } \\
\text { benignas }\end{array}$ & 78 & 8,3 \\
\hline $\begin{array}{l}\text { Lesões proliferativas neoplásicas } \\
\text { malignas }\end{array}$ & 25 & 2,7 \\
\hline Lesões ulceradas da mucosa bucal & 46 & 4,9 \\
\hline Lesões vermelhas da mucosa bucal & 106 & 11,3 \\
\hline Patologias ósseas & 18 & 1,9 \\
\hline Outras classificações & 34 & 3,5 \\
\hline Tumores odontogênicos & 16 & 1,7 \\
\hline Total & 940 & 100 \\
\hline
\end{tabular}

Com relação ao diagnóstico clínico das lesões, a Tabela 3 demonstra os valores dos dezesseis diagnósticos mais prevalentes nos prontuários consultados.

Tabela 3 - Distribuição dos dezesseis diagnósticos clínicos mais prevalentes para as lesões estudadas

\begin{tabular}{l|r|r}
\hline \multicolumn{1}{c|}{ Diagnóstico clínico } & № & \multicolumn{1}{c}{$\%$} \\
\hline Fibroma de irritação & 142 & 15,1 \\
Candidíase & 71 & 7,6 \\
Hiperplasia fibrosa dos sulcos & 69 & 7,3 \\
Mucocele & 68 & 7,2 \\
Líquen plano & 52 & 5,5 \\
Candidíase atrófica & 45 & 4,8 \\
Queilite actínica & 40 & 4,3 \\
Hemangioma & 37 & 3,9 \\
Úlcera traumática & 30 & 3,2 \\
Língua geográfica & 26 & 2,8 \\
Granuloma piogênico & 24 & 2,6 \\
Carcinoma epidermoide & 20 & 2,1 \\
Síndrome da ardência bucal & 19 & 2,0 \\
Queratose friccional & 17 & 1,8 \\
Hiperplasia papilar inflamatória & 14 & 1,5 \\
Papiloma escamoso & 14 & 1,5 \\
\hline & & \\
\hline
\end{tabular}

Quanto à área geográfica de origem dos pacientes, o presente estudo envolveu indivíduos provenientes de 114 municípios, de quatro macrorregiões sul-rio-grandenses (Norte, Metropolitana, Serra e Missioneira), dentre as sete macrorregiões do estado do Rio Grande do Sul (conforme dados do Instituto Brasileiro de Geografia e Estatística), que abrangem 497 municípios. As dezessete cidades de origem dos pacientes mais atendidos na FOUPF (Tabela 4) foram: Passo Fundo, Soledade, Camargo, Sarandi, Marau, Coqueiros do Sul, Tapejara, Carazinho, Chapada, Palmeira das Missões, Engenho Velho, Lagoa dos Três Cantos, Santa Cecília do Sul, Santo Antônio do Planalto, Constantina, Lagoa Vermelha e Pontão.

Tabela 4 - Distribuição dos tratamentos realizados

\begin{tabular}{l|r|r}
\hline \multicolumn{1}{c|}{ Cidades } & $\begin{array}{r}\text { Frequência } \\
\text { (n) }\end{array}$ & \multicolumn{1}{c}{$\%$} \\
\hline Passo Fundo & 190 & 30,9 \\
Soledade & 39 & 4,1 \\
Camargo & 24 & 2,6 \\
Sarandi & 23 & 2,4 \\
Marau & 22 & 2,3 \\
Coqueiros do Sul & 21 & 2,2 \\
Tapejara & 21 & 2,2 \\
Carazinho & 20 & 2,1 \\
Chapada e Palmeira das Missões & 16 & 1,7 \\
Engenho Velho, Lagoa dos Três & 14 & 1,5 \\
Cantos, Santa Cecília do Sul e & & \\
Santo Antônio do Planalto & 13 & 1,4 \\
Constantina, Lagoa Vermelha e & & \\
Pontão & 940 & 100 \\
Total &
\end{tabular}

\section{Discussão}

A epidemiologia foi definida por Last $^{5}$ (2001) como "o estudo da distribuição e dos determinantes de estados ou eventos relacionados à saúde em populações específicas, e sua aplicação na prevenção e controle dos problemas de saúde". Essa definição deixa claro que os epidemiologistas estão preocupados não somente com a doença, mas também com a melhoria dos indicadores de saúde e com maneiras de promover saúde.

Rouquayrol e Almeida Filho ${ }^{6}$ (1994) definem claramente a importância da realização de levantamentos epidemiológicos quando se trata de saúde. É por meio de levantamentos que se pode analisar a distribuição e os fatores determinantes de doenças, propondo medidas específicas de prevenção, controle e erradicação. Além disso, tais levantamentos fornecem indicadores que podem servir para o planejamento de ações em saúde.

Qualquer que seja a especialidade na qual o cirurgião-dentista atue, o exame sistemático da boca é de suma importância. A odontologia, como profis- 
são de saúde, não se restringe apenas ao cuidado dos dentes e de suas estruturas de suporte, mas também se enquadra atualmente na área de prevenção e diagnóstico de doenças da mucosa bucal.

No presente estudo, conforme os resultados obtidos, pode-se notar que uma amostra relevante foi analisada, por terem sido avaliados 18.801 prontuários do período entre 2000 e 2013 , e terem sido encontradas 940 lesões bucais, diferentemente de muitos trabalhos publicados, como os de Mathew et al. ${ }^{7}$ (2008), que fizeram o levantamento em apenas 1.190 prontuários, ou o de Vieira et al. ${ }^{8}$ (2006), que analisaram 215 pacientes.

$\mathrm{Na}$ amostra do presente estudo, em relação ao gênero, observou-se que o mais prevalente foi o feminino $(64,5 \%)$, dados que corroboram os estudos de Cardoso et al. ${ }^{9}$ (2005), Vieira et al. ${ }^{8}$ (2006), Rocha et al. ${ }^{10}$ (2006), Bertoja et al. ${ }^{11}$ (2007), Mujica et al. ${ }^{12}$ (2008), Henrique et al. ${ }^{13}$ (2009), Casagrande ${ }^{14}$ (2011), Vaz et al..$^{15}$ (2011), Suzin et al. ${ }^{3}$ (2014) e Souza et al. ${ }^{4}$ (2014). Esse resultado pode ser explicado em razão da expressiva procura das mulheres por centros de saúde odontológicos e também por elas se submeterem mais aos exames dentários de rotina, demostrando mais preocupação em relação à saúde. Ao se aplicar o teste Qui-Quadrado $\left(\mathrm{x}^{2}\right)$ aos resultados obtidos, notou-se significância estatística $(<0,05 \%)$, o que comprova ser o gênero feminino o mais expressivo no estudo.

Com relação à etnia da amostra estudada, a mais acometida foi a leucoderma, com $90 \%$ dos casos, resultado semelhante aos achados de De Carli et al. ${ }^{16}$ (2009), Henrique et al. ${ }^{13}$ (2009) e Souza et al. $^{4}$ (2014).

Quando analisada a idade dos pacientes, encontrou-se uma prevalência na quarta, quinta e sexta décadas de vida $(61,2 \%)$, sendo a quinta década a mais prevalente, com média de idade de 47,55 anos. A amostra estudada variou de 1 a 88 anos de idade, e a faixa etária de menor prevalência foi de 80 a 88 anos, com 0,7\% ( $\mathrm{n}=7)$. Esses dados divergem dos achados de Vieira et al. ${ }^{8}$ (2006), Bertoja et al. ${ }^{11}$ (2007) e Vaz et al..$^{15}$ (2011), que verificaram média de idade de, respectivamente, 33,1 anos, 44,2 anos e 36,12 anos. Em contrapartida, os achados de Rocha et al. $^{10}$ (2006), Cornejo et al. ${ }^{17}$ (2007), De Carli et al. ${ }^{16}$ (2009) e Suzin et al. ${ }^{3}$ (2014) confirmam o acometimento das décadas mais prevalentes no presente estudo.

Conforme verificado no presente trabalho, os pacientes entre a quarta e a sexta décadas de vida são os que mais procuram atendimento nas clínicas odontológicas da FOUPF, o que, muitas vezes, se deve ao fato de esses pacientes serem portadores de próteses, o que aumenta a chance do aparecimento de lesões proliferativas hiperplásicas (29\% no total). Desse modo, as lesões mais frequentes nesse grupo foram os fibromas de irritação (142 casos $-15,1 \%$ ), as hiperplasias fibrosas dos sulcos (69 casos - 7,3\%), os granulomas piogênicos (24 ca- sos $-2,6 \%$ ) e as hiperplasias papilares inflamatórias ( 14 casos $-1,5 \%)$. Tais achados se equiparam aos de Rocha et al. ${ }^{10}$ (2006), Moresco et al. ${ }^{18}$ (2003), Bertoja et al. ${ }^{11}$ (2007), Vaz et al. ${ }^{15}$ (2011) e Mujica et al. ${ }^{12}$ (2008), que obtiveram, nos casos de fibroma, $34,9 \%, 54,2 \%, 21,2 \%, 12,7 \%$ e $7 \%$, respectivamente. De outro modo, Mathew et al. ${ }^{7}$ (2008) encontraram somente $0,84 \%$ para os casos de fibroma de irritação.

Em relação às lesões brancas ceratóticas da mucosa bucal (14,9\%), o líquen plano (52 casos - 5,5\%) foi o mais prevalente, dado que vem ao encontro da pesquisa feita por Axell $^{19}$ (1978) e diverge de Mathew et al. $^{7}$ (2008). No grupo das lesões vermelhas da mucosa bucal (11,3\%), a candidíase atrófica (45 casos $-4,8 \%$ ) aparece em primeiro lugar e foi a que mais acometeu os pacientes pertencentes a esse grupo. Tais dados não foram encontrados em nenhuma amostra da revisão de literatura do presente trabalho. A candidíase $(71$ casos $-7,6 \%$ ) foi a que mais prevaleceu no grupo das lesões brancas não ceratóticas da mucosa bucal, igualmente aos estudos de Mathew et al. ${ }^{7}(2008)$ e Henrique et al. ${ }^{13}(2009)$. Em relação ao hemangioma (37 casos $-3,9 \%$ ), pertencente ao grupo das lesões proliferativas neoplásicas benignas $(8,3 \%)$, os resultados do presente estudo assemelham-se aos achados da pesquisa feita por Mujica et al. ${ }^{12}$ (2008), em que os casos de hemangioma apareceram com $11 \%$, e aos de Suzin et al. ${ }^{3}$ (2014), com $52,71 \%$ (146 casos).

$\mathrm{Na}$ amostra do presente estudo, pode-se notar que as lesões relacionadas ao fungo Candida albicans estiveram presentes nos casos de candidíase (71 casos), candidíase atrófica (45 casos), candidíase pseudomembranosa (11 casos), hiperplasia papilar inflamatória (14 casos) e glossite romboidal mediana ( 2 casos), totalizando 143 casos (15,3\%). Tais dados corroboram os achados de Mathew et al. ${ }^{7}$ (2008) e Henrique et al. ${ }^{13}$ (2009).

Com relação à procedência dos pacientes estudados, observou-se que aqueles que procuraram atendimento na área de estomatologia da FOUPF o fizeram após indicação de cirurgiões-dentistas particulares ou das unidades básicas de saúde, que encaminharam esses pacientes quando havia alguma dúvida no diagnóstico. Pacientes da região Norte do Rio Grande do Sul foram predominantes pelo fato de a Faculdade de Odontologia da Universidade de Passo Fundo estar situada nessa área geográfica. Os pacientes eram oriundos de Passo Fundo $(30,9 \%)$, Soledade $(4,1 \%)$, Camargo $(2,6 \%)$, Sarandi $(2,4 \%)$ e Marau (2,3\%). Observou-se que os pacientes eram procedentes de 114 municípios, compreendidos em quatro macrorregiões sul-rio-grandenses (Norte, Metropolitana, Serra e Missioneira), dentre as sete existentes no estado.

A amostra estudada reflete a importância da Universidade de Passo Fundo como um centro de referência de diversos serviços, além de evidenciar a capacidade de atendimento com qualidade de um 
grande número de pacientes odontológicos, visto que todos receberam atendimento e tratamento completos.

\section{Conclusão}

De acordo com os dados obtidos na análise dos prontuários da FOUPF do período entre 2000 e 2013, observaram-se diversos tipos de lesões bucais, sendo as mais frequentes: fibroma de irritação, candidíase, hiperplasia fibrosa dos sulcos, mucocele e líquen plano. $\mathrm{O}$ perfil epidemiológico do paciente atendido no período estudado foi composto predominantemente por mulheres com mais de quarenta anos de idade e por leucodermas.

Diante deste estudo, pode-se afirmar que a Faculdade de Odontologia da Universidade de Passo Fundo conta com uma excelente estrutura, tanto pelo número de pacientes que a frequenta como pelos resultados ora apresentados, sendo capaz de proporcionar atendimento e resolução de problemas, além de boa coleta e armazenamento de dados. Isso permite que diversos outros estudos de importância sejam realizados nessa universidade, como estudos prospectivos de grande população, análises a partir do conhecimento do perfil da população atendida no serviço e estudos comparativos entre amostras de diferentes épocas. Percebeu-se o importante papel que o cirurgião-dentista desempenha no exame adequado das áreas anatômicas da boca e anexos, de modo a reconhecer, identificar, diagnosticar e realizar tratamentos adequados para restabelecer a saúde bucal dos pacientes.

\section{Abstract}

The importance of conducting epidemiological studies, as well as defining the prevalence of lesions found in a given geographical area, is to establish preventive methods and promote oral health. Objective: This study aimed to perform an epidemiological survey of the most frequent oral lesions from dental records of the School of Dentistry of the University of Passo Fundo (FOUPF), RS, Brazil, from 2000 to 2013. Materials and method: 18,801 medical records were analyzed. From the data collected, 940 lesions were found and analyzed with the SPSS version 15.0 software. Results: The five most prevalent lesions were irritation fibroma (15.1\%), candidiasis (7.6\%), fibrous hyperplasia of sulcus (7.3\%), mucocele $(7.2 \%)$, and lichen planus (5.5\%). Women were most affected $(6.5 \%)$, and the most prevalent ethnicity was Caucasian (90\%). The group of proliferative hyperplastic lesions was most prevalent (29\%), followed by the non-keratotic white lesions (14.9\%), red lesions of the oral mucosa (11.3\%), non-keratotic white lesions (9\%), and benign proliferative neoplastic lesions (8.3\%). It was found that the age group between 40 and 69 years was most affected (61.2\%). Conclusions: It was observed in the present study that because the FOUPF is located in a geographical area of easy access and reference in the health field, both for medical and dental care, the demand for care was major for Northern and Northwest Rio Grande do Sul, thus allowing to outline an epidemiological profile of patients most affected by oral lesions.

Keywords: Oral Medicine. Epidemiology. Oral lesions.

\section{Referências}

1. Antunes JLF, Peres MA. Epidemiologia da saúde bucal. Rio de Janeiro: Guanabara Koogan; 2006. 470 p.

2. Cebeci ARI, Gïlsahi A, Kamburoglu K, Orhan BK, Öztas B. Prevalence and distribution of oral mucosal lesions in an adult Turkish population. J Med Oral Patol Oral Cir Bucal $2009 ; 6(1): 272-7$.

3. Suzin TL, Frigo TZ, Salum FG, Cherubini K, Figueiredo MAZ. Levantamento epidemiológico em portadores de patologias de língua atendidos no Serviço de Estomatologia e Prevenção do Câncer Bucomaxilofacial do Hospital São Lucas da Pontifícia Universidade Católica do Rio Grande do Sul (PUCRS): estudo retrospectivo. Rev Odontol Bras Central 2014; 23(64):14-7.

4. Souza JGS, Soares LA, Moreira G. Concordância entre os diagnósticos clínicos e histopatológicos de lesões bucais diagnosticadas em Clínica Universitária. Rev Odontol Unesp 2014; 43(1):30-5

5. Last JM. A dictionary of epidemiology. 4. ed. Oxford: Oxford University Press; 2001.

6. Rouquariol MZ, Almeida Filho N. Epidemiologia e Saúde. 4. ed. Rio de Janeiro: Medsi; 1994.

7. Mathew AL, Pai KM, Sholapurkar AA, Vengal M. The prevalence of oral mucosal lesions in patients visiting a dental school in Southern India. J Dent Res 2008; 19:99-103.

8. Vieira VG, Fernandes AM, Machado APB, Grossman SMC, Aguiar MCF. Prevalência das alterações da normalidade lesões da mucosa bucal em pacientes atendidos nas Clínicas Integradas de Atenção Primária (Ciaps) da Faculdade de Odontologia da UFMG. Arq Odontol 2006; 42(4):257-336.

9. Cardoso SO, Silva SS, Queiroga J, Leão JC. Avaliação da prevalência de grânulos de Fordyce em 2.281 indivíduos da cidade do Recife. Rev Fac Odontol Porto Alegre 2005; 46(1):25-9.

10. Rocha DAP, Oliveira LMM, Souza LB. Neoplasias benignas da cavidade oral: estudo epidemiológico de 21 anos (1982 a 2002). Rev Odontol Univ Cidade de São Paulo 2006; 18(1):53-60.

11. Bertoja IC, Tomazini JG, Braosi APR, Luis JCZ, Reis FG, Giovanini AF. Prevalência de lesões bucais diagnosticadas pelo Laboratório de Histopatologia do UnicenP. RSBO 2007; 4(2):34-7.

12. Mujica V, Rivera H, Carrero M. Prevalence of oral soft tissue lesions in an elderly Venezuelan population. Rev Med Oral Patol Oral Cir Bucal 2008; 13(5):18-22.

13. Henrique PR, Bazaga Júnior M, Araújo VC, Junqueira JLC, Furuse C. Prevalência de alterações da mucosa bucal em indivíduos adultos da população de Uberaba, Minas Gerais. Rev Gaúcha Odontol 2009; 57(3):261-7.

14. Casagrande FB. Diagnóstico e levantamento epidemiológico de doenças do complexo maxilo-mandibular no centro de especialidades odontológicas (CEO) Jardim Periperi (Butantã). São Paulo: Universidade Municipal de São Caetano do Sul; 2011.

15. Vaz DA, Valença DL, Lopes RBM, Costa e Silva AV, Pereira JRD. Concordância entre os diagnósticos clínicos e histopatológicos do Laboratório de Patologia Bucal da Faculdade 
de Odontologia de Pernambuco. RPG Rev Pós Grad 2011; 18(8):236-43.

16. De Carli ML, Santo SL, Pereira AAC, Hanemmann JAC. Características clínicas, epidemiológicas e microscópicas do câncer bucal diagnosticado na Universidade Federal de Alfenas. Rev Bras Cancerol 2009; 55(3):64-9.

17. Cornejo AD, Huerta ERL, Bravo SP, Barrios BA, Rivera DQ, Yañez AB et al. Distribución de condiciones y lesiones de la mucosa bucal en pacientes adultos mexicanos. Rev Cubana Estomatol 2007; 44(1):25-32.

18. Moresco FC, Nora Filho MR, Balbinot MA. Levantamento epidemiológico dos diagnósticos histopatológicos da disciplina de Estomatologia da Faculdade de Odontologia da Ulbra - Canoas/RS. Rev Stomatos 2003; 9(17):29-34.

19. Axell T. A prevalence study of oral mucosal lesions in an adult Swedish population. Odontol Revy Suppl 1978; 36:1103

\section{Endereço para correspondência:}

Soluete Oliveira da Silva

Rua Padre Nóbrega, 475/301, Lucas Araújo

99070-140 Passo Fundo-RS

Fone: (54) 3312-3800

E-mail: soluete@upf.br

Recebido: 21/09/15. Aceito: 20/10/15. 